\title{
Is the pitching moment acting on a Javelin always nose-down?
}

Kazuya Seo ( $\nabla$ seo@sci.kj.yamagata-u.ac.jp )

Yamagata University

Hiroyuki Okuizumi

Tohoku University

Yasufumi Konishi

Tohoku University

Takuto Kobayashi

Yamagata University

Hiroaki Hasagawa

Utsunomiya University

Shigeru Obayashi

Tohoku University

\section{Research Article}

Keywords:

Posted Date: February 16th, 2022

DOI: https://doi.org/10.21203/rs.3.rs-1319335/v1

License: (c) (i) This work is licensed under a Creative Commons Attribution 4.0 International License.

Read Full License 


\section{Abstract}

The rules governing the dimensions of the Javelin were substantially changed in 1986. It was considered that this new design guaranteed there was zero pitching moment at $0^{\circ}$ angle of attack and that the pitching moment decreased (became negative) with increasing angle of attack. The objective of this study is to investigate if the pitching moment remains always negative (nose-down rotation). To measure accurate aerodynamic forces acting on a Javelin, the world's largest $1 \mathrm{~m}$ magnetic suspension and balance system was used. The magnetic suspension and balance system was able to measure aerodynamic forces without support interference in the wind tunnel. In addition, computational fluid dynamics were carried out to estimate the pitching moment coefficients. It was found that the pitching moment coefficient of a commercially available Javelin becomes positive (nose-up rotation) at lower angles of attack, less than $12^{\circ}$. The pitching moment becomes positive if the upstream side of the center of gravity receives more inflow than the downstream side. This situation can be attained by, for example, increasing the thickness of the upstream side when compared with that of the downstream side.

\section{Introduction}

A conventional wind tunnel test for a Javelin would be performed by fixing the Javelin to a supporting rod 1,2 . However, the supporting rod disturbs the flow and this is known as support interference. For example, the separation line on the ellipsoid has been observed to drastically move backwards when using a thin rod whose diameter is $0.5 \mathrm{~mm}^{3}$. Generally, in the case of the equipment of sports, the sizes are comparable with hands or feet. In particular, for a long and narrow object like the javelin, support interference becomes significant because the diameter of the supporting rod is comparable to that of the javelin, making it difficult to measure the aerodynamic forces accurately 4,5 .

A Magnetic Suspension and Balance System (MSBS) is a valuable tool for measuring aerodynamic forces without support interference. The first MSBS was developed in ONERA in 1950s ${ }^{6}$. However, further research and development of this MSBS has been suspended since 1970s. This was because there was no prospect of commercialization of MSBS ${ }^{7}$. Nowadays, research and development of MSBS has been restarted in ODU ${ }^{8}, \mathrm{KAIST}^{9}$ and Tohoku University ${ }^{10}$ due to improved sophistication of the measuring equipment and improved computer control, as well as the development of powerful neodymium magnets. However, there are still relatively few MSBS's in the world.

From the view of aerodynamics of the Javelin, the rules governing the dimensions were substantially changed in 1986. The main factor that motivated the change was that in many throws the Javelin was landing nearly flat, causing difficulties for the judges to determine whether the throw was valid or not ${ }^{11}$. It was considered that the new design guaranteed that the pitching-moment profile of the Javelin was monotonically decreasing with increasing the angle of attack, without ever attaining a positive value.

The objective of this study is to make sure whether the pitching moment is always negative (nose-down rotation) with respect to the angle of attack. The aerodynamic forces acting on the Javelin without the 
supporting rod will be described. The world's largest magnetic suspension and balance system (MSBS) was employed to measure the aerodynamic forces acting on a full-size women's Javelin. Therefore, the aerodynamic coefficients shown in this paper should be the most accurate. In addition, a parametric study using CFD (Computational Fluid dynamics) was also carried out assess whether the pitching moment is always negative.

\section{Methods}

Principle of the Magnetic Suspension and Balance System. The working principle of the magnetic suspension and balance system (MSBS) is shown in Figure 1. A Javelin which includes magnets along the longitudinal axis is levitated at the center of the test section. When wind flows, aerodynamic forces act on the Javelin and the control principle is designed to keep the Javelin at the center of the test section (home position). To keep the same position and the same attitude of the Javelin, ten coils are placed around the test section. The current of the coils is adjusted to keep the same position and the same attitude. The drive current differences between the wind-on condition and the wind-off condition are converted into aerodynamic forces.

Javelin. A commercially available full-size women's Javelin (Hybrid Genome X, Nishi) was employed for the wind tunnel tests. The length of the Javelin was $2210 \mathrm{~mm}$, and the center of gravity was at $920 \mathrm{~mm}$ from the tip. Most of the surface of the Javelin was spray painted white, as shown in Figure 2-(a), to aid detecting the position. For the same reason, a 15-mm-long collar was attached at the center of gravity, and 5-mm-wide black tape was wrapped around the Javelin at the center of gravity. Neodymium magnets were inserted along the longitudinal axis, as shown in Figure 2-(b). Two types of the magnet with diameters of $19 \mathrm{~mm}$ and $20 \mathrm{~mm}$ were used, and the total length of the magnet assembly was $495 \mathrm{~mm}$. Grip strings were wound around the javelin both on the upstream and downstream sides of the collar as shown in Figure 2-(c). The diameter of the strings, which is $4 \mathrm{~mm}$, has the same width as the height of the 15 -mm-long collar.

Wind tunnel. The low turbulence wind tunnel facility at the Institute of fluid science, Tohoku university was used for our work ${ }^{12}$. Since the distance between opposite sides of the hexagonal bell-mouth is 1.01 $\mathrm{m}$, it was possible to employ a full-size women's javelin in a wide range of angle of attack $(A \circ A)$ up to $18^{\circ}$. The wind tunnel turbulence levels are among the lowest (less than $0.02 \%$ at $25 \mathrm{~m} / \mathrm{s}$ ) in the world. Moreover, the uniformity of its velocity profiles is within $\pm 0.02 \%$ with respect to the average velocity, making it possible to conduct very high-quality aerodynamics research. The experimental results presented in this paper would be expected to be very accurate because of the use of the MSBS without supporting interference and using such a large and low turbulence wind tunnel.

Position-sensing system. A schematic of the optical position-sensing system is shown in Figure 3-(a). The coordinate system is also shown. The origin was at the center of gravity of the javelin, with the positive $x$-axis in the horizontal upstream direction, the $y$-axis was also horizontal and orthogonal to the $x$ axis. The positive $z$-axis was vertically upward. The optical position-sensing system is composed of a 
convex lens (focal length $125 \mathrm{~mm}$ ), dichroic color filters (red and blue), a half mirror, red and blue LED lights (MSPP-CB74, Moritex), and position sensors which are CCD (Charge-Coupled Device) line sensor camera (TL7450S, Takenaka system equipment). CCD line sensor camera is composed of 7450 CCDs in a line. The size of the CCD element is $4.7 \mu \mathrm{m}$ times $4.7 \mu \mathrm{m}$, and the pixel resolution is less than $10 \mu \mathrm{m}$. The sampling frequency is $1250 \mathrm{~Hz}$.

Position calibration. The real position and attitude were defined by the five-component stages (ALS904H1P, ALV-104HP, ATS-130HP \& ARS-936HP, Central Motor Wheel). The position sensors were calibrated with the defined position and attitude. An example of the calibration results in the $x$-axis are shown in Figure 3-(b). In this case, the five-component stages were moved only in the $x$-axis. The output value from the position sensor on the $x$-axis varies linearly with respect to the real position change in the $x$-axis. Since the five-component stages moved in the only $x$-axis, the output counting value in the $y$-axis did not change (insensitive in the $y$-axis). Other axes were calibrated in the same manner.

Levitation of the javelin and use of a notch filter. The first trial to levitate the Javelin failed. The time variations in the $y$-direction are shown in Figure 4-(a). The javelin was unstable and diverged from its initial position after only $0.25 \mathrm{~s}$. The frequency components observed were $22 \mathrm{~Hz}$ and $55 \mathrm{~Hz}$. The frequency of $22 \mathrm{~Hz}$ corresponded to the principle resonant frequency of the javelin ${ }^{13,14}$ and was the primary reason we were unable to control it. Therefore, a notch filter (band-stop filter) was employed to cut out the resonant frequency. As can be seen in Figure 4-(b), the notch filter stabilized the Javelin, allowing us to levitate the javelin in the MSBS.

In principle, the javelin should be always stabilized at the same position and the same attitude in the MSBS. However, the presence of the resonance enabled us to realize a vibrating model in the MSBS with the resonance frequency of the Javelin. Figure 4-(c) shows the time variation of $y$-direction with a weak notch filter, i.e., a filter with decreased intensity. By decreasing the intensity of the notch filter, vibration of the Javelin, as observed in real flight, was realized. The frequency of the vibration was $22 \mathrm{~Hz}$, as before, but the Javelin remained under control. Figure 5 shows the javelin levitated in the test section in the wind tunnel. It was illuminated brightly around the center of gravity to detect the position. The AoA is $18^{\circ}$, which is the largest value we can use in the world's largest MSBS. This is because the tail of the javelin is approaching the wall of the test section, but still outside of the boundary layer of the wall at $18^{\circ}$ and because the LED lighting to detect the position can't illuminate the Javelin at more than $18^{\circ}$. The $A o A$ was changed in the horizontal plane (on the vertical $z$-axis). This definition of $A \circ A$ allowed us to decrease the current when compared with the change of $A O A$ in the vertical plane.

Force calibration. To relate the forces to the current, several weights were applied as calibration references. For example, Figure 6-(a) shows the schematic of force calibration in the $x$-axis. The weights, $F_{x}$, were applied to the levitated javelin only in the $x$-axis via jig and pulleys. The calibration results are shown in Figure 6-(b). The current applied in the $x$-axis, $l_{x}$, increases linearly with increasing $F_{x}$. The force in the $y$-axis and the moment on the $z$-axis were calibrated in the same manner. 
Computational Fluid Dynamics (CFD). The simulation was carried out using ANSYS 2021 R1, Design Modeler, Meshing and Fluent. A commercially available full-size women's javelin (Hybrid Genome X, Nishi) was drawn using Design modeler. The length is $2.21 \mathrm{~m}$, whilst the maximum diameter is $0.0247 \mathrm{~m}$. The dimensions of the computational domain (enclosure) are $600 \mathrm{~m} \times 600 \mathrm{~m} \times 10 \mathrm{~m}$ in the vertical and lateral directions respectively.

Meshing was used for the computational domain. The summary is shown in Table 1. A first layer thickness inflation option was adopted to create an inflation mesh structure. The maximum skewness is about 0.89. Figure 7 shows meshes around the javelin (Figure 7-(a)) and the javelin's top (Figure 7-(b)). Fluent was used to solve the 3D Reynolds-Average Navier-Stokes (RANS) equations and the continuity equation, using the finite volume method. A summary is also shown in Table 1. A standard $k-\varepsilon$ model with standard wall functions is used for turbulence modeling.

Table 1

Summary of meshing and boundary conditions.

\begin{tabular}{|lll|}
\hline Set-up & Variable & Settings \\
\hline Mesh & Element size & $150[\mathrm{~mm}]$ \\
\hline & Nodes & $3,703,025$ \\
\hline & Elements & $20,329,462$ \\
\hline Max skewness & 0.89 \\
\hline Inflation & First layer thickness & $2.0[\mathrm{~mm}]$ \\
\hline Turbulent model & & Standard k- $\varepsilon$ \\
\hline Boundary condition & In: Velocity-inlet & $25[\mathrm{~m} / \mathrm{s}]$ \\
\hline & Out: Pressure-outlet & Gauge pressure:0 [Pa] \\
\hline & Wall, Surface of javelin & Non-slip \\
\hline
\end{tabular}

\section{Results}

Experimental results of aerodynamic forces acting on a javelin. The time averaged aerodynamic coefficients, $C_{D}, C_{L}$ and $C_{m}$ on the static and the dynamic javelin are shown as a function of the angle of attack, $A \circ A$ in Figure 8. The $A \circ A$ of the dynamic javelin is also the time averaged value, which is defined at $150 \mathrm{~mm}$ each on the upstream and downstream sides of the center of gravity. The $95 \%$ confidence intervals are also shown as error bars. The $C_{D}$ and $C_{L}$ are defined by equation (1) and (2), while the $C_{m}$ is defined by equation (3).

$C_{D}=\frac{D}{0.5 \rho U^{2} A}(1)$ 
$C_{L}=\frac{L}{0.5 \rho U^{2} A}(2)$

$C_{m}=\frac{M}{0.5 \rho U^{2} A l}(3)$

Here, $\rho$ is the air density, $U$ is the wind speed, $A$ is the cross-sectional area at the maximum diameter $(0.0247 \mathrm{~mm})$, / is the length of the javelin $(2.21 \mathrm{~m})$.

The $C_{D}$ and $C_{L}$ for both cases increase with increasing $A O A$ in the range between 0 and $18^{\circ}$. The error bars are small, that is, the measured data are very repeatable. The $C_{m}$ becomes positive up to $10^{\circ}$. The $C_{m}$ for both cases increases until $8^{\circ}$, then it decreases above that angle. The error bars are smaller at lower $A \circ A$. The value becomes negative above $12^{\circ}$ and the absolute value increases with increasing $A O A$. When it becomes negative, the error bar becomes large and the date less repeatable. Longitudinal static stability concerned with the pitching moment occurs around about $12^{\circ}$. The $C_{L}$ and $C_{m}$ are almost 0 at $0^{\circ}$. The aerodynamic coefficients of the dynamic javelin are almost comparable with those of the static javelin.

CFD results. The aerodynamic coefficients acting on the static javelin are shown in Figure 9. The experimental fluid dynamics results (EFD (Static case)) are measured from the MSBS, while the computational fluid dynamics (CFD) results are calculated by Ansys Fluent. The EFD results in Figure 8 are shown again. The $C_{D}$ data obtained by CFD quantitatively agree with those by EFD, while the $C_{L}$ and $C_{m}$ obtained by CFD qualitatively agree with those by EFD. Therefore, the CFD can effectively simulate the aerodynamic coefficients on the javelin.

The $C_{m}$ on the static javelin obtained by CFD for three cases are shown in Figure 10. The difference amongst these three is the thickness of the top of the javelin. The thickness was changed within the range of the rules governing the dimensions of the javelin. It was found that the modern javelin still has positive values of $C_{m}$ at lower $A o A$. In the case of the thinner top, the pitching-moment profile was monotonically decreasing with increasing $A O A$, without ever attaining a positive value as described in reference 11.

\section{Discussion}

It was found that the modern Javelin has positive values of $C_{m}$ (nose-up) at lower $A o A$, as seen in Figure 8(c) and Figure 10. Consideration of whether the pitching moment is positive or not depends on the force difference between the upstream side and the downstream side of the center of gravity. If the force acting on the upstream side is larger than that on the downstream side at a certain $A \circ A$, a positive pitching moment around the center of gravity acts on the Javelin. Since the center of gravity of the Javelin is located at the upstream side from the geometric center, the pitching moment, in principle, tends to be negative. However, the pitching moment becomes positive, if the upstream side of the center of gravity gets more inflow than the downstream side. This situation can be attained by, for example, increasing the thickness on the upstream side when compared with that of the downstream side as shown in Figure 10. 
The key to attain longer flight distances is to decrease the drag in the first half and to increase the lift in the second half of the flight ${ }^{15,16}$. These are attained with a pitching-moment profile like Figure 8-(c).

It has been reported that the vibrations of the javelin increase both lift and drag ${ }^{13}$. We observe that the aerodynamic coefficients of the dynamic (vibrating) javelin are almost comparable with those of the static Javelin, as shown in Figure 8. Figure 8-(a) shows that the $C_{D}$ of the dynamic case is slightly larger than that of the static case at lower $A O A$ as predicted by previous simulations ${ }^{13}$, but the difference is not as large as predicted in the previous simulations. On the other hand, Figure 8-(b) shows that the $C_{L}$ of the dynamic case is slightly smaller than that of the static case. This is an opposite effect of the vibration to that predicted in reference 13. This inconsistency between the previous simulation and the present data may occur because of the model assumed in reference 13 . The relative velocity with respect to the Javelin was defined as the sum of the main inflow velocity and the velocity caused by the vibration. The main inflow against the javelin was assumed to be same over the longitudinal axis. However, the speed of main inflow with respect to the downstream side might be decreased in the wake, especially at lower $A \circ A$. As a result, the aerodynamic forces acting on the dynamic Javelin in the wake might be smaller when compared with the previous simulation.

In future studies, the deformation of the Javelin should be measured simultaneously with the aerodynamic forces acting on the vibrating javelin. Moreover, a Bayesian optimization of the flight distance in consideration of the precise shape and stiffness of the Javelin in which the fluid-structure interaction problems are considered should be carried out.

\section{Conclusion}

To accurately measure the aerodynamic forces acting on a Javelin without support interference, the world's largest magnetic suspension and balance system was used. By utilizing a notch filter (band-stop filter for the resonance frequencies) and varying its intensity, both the static and dynamic (vibrating) cases were measured. It was found that the modern Javelin has positive values of the pitching moment coefficients (nose-up) at lower angles of attack. The pitching moment becomes positive, if the upstream side of the center of gravity gets more inflow than the downstream side. The time averaged aerodynamic forces of the dynamic case were comparable to those of the static case.

\section{Declarations}

\section{Competing interests (mandatory)}

The authors declare no competing interests.

\section{Acknowledgements (optional)}

The authors would like to thank Kaori Sasaki, Shinya Shinji, Shogo Oyama, Tohoku University, for their support. 
This research was funded by JSPS KAKENHI, grant number $19 \mathrm{H} 05570$.

\section{Author contributions}

K.S., H.O., Y.K., H.H. and S.O. conceived the experiments. K.S., H.O., Y.K. and T.K. conducted the experiments. K.S., H.O. and T.K. performed the statistical analysis and figure generation, and K.S. wrote the main manuscript text. All authors reviewed the manuscript.

\section{References}

1. Chowdhury, H., Alam, F., Muscara, A. \& Mustary, I. An experimental study of new rule javelins. Procedia Engineering, 60, 485-490 (2013).

2. Terauds J. Wind tunnel tests of competition javelins. Track and Field Quarterly Review, 74, 88-95 (1974).

3. Ambo, T., Nakamura, Y., Ochiai, T., Nonomura, T. \& Asai, K. Visualization of Flow Separation on a Prolate Spheroid by using a Magnetic Suspension and Balance System. Fourteenth International Conference on Flow Dynamics, Sendai, Japan, Nov. 1st -3rd, (2017).

4. Sasaki, K. Aerodynamic Measurements of Turbo-Jav Model Using 1-m Magnetic Suspension and Balance System. Master thesis, Tohoku univ. (2020) (in Japanese).

5. Kobayashi, T., Seo, K., Kaneda, S., Sasaki, K., Shinji, K., Oyama, S., Okuizumi, H., Konishi, Y., Hasegawa, H. \& Obayashi, S. Measurement of the Aerodynamic Forces Acting on a Non-Spinning Javelin Using an MSBS. Proceedings, 49, 144 (2020). https://doi.org/10.3390/proceedings2020049144.

6. Chrisinger, J. E., Tilton III, E. L., Parkin, W. J., Cofin J. B. \& Covert, E. E. Magnetic Suspension and Balance System for Wind Tunnel Application. J. Royal Aeronaut. Soc., 67, 717-724 (1963).

7. Sawada, H. \& Kunimasu, T. Development of a $60 \mathrm{~m}$ Magnetic suspension and Balance system. J. of the Japan Soc. for Aeronautical and Space Sci. 50, 188-195 (2002) (in Japanese).

8. Schott, T., Cox, D., Schoenenberger M. \& Miller F. Operational Experience with the Electromagnetic Position Sensor (EPS) for the NASA 6-inch MSBS. AIAA Scitech 2021 Forum, January, AIAA 20211872 (2021). https://doi.org/10.2514/6.2021-1872.

9. Sung, Y-H, Lee D-K, Han J-S, Kim H-Y \& Han J-H. MSBS-SPR Integrated System Allowing Wider Controllable Range for Effective Wind Tunnel Test. Int. J. of Aeronaut. and Space Sci., 18, 414-424 (2017). https://doi.org/10.5139/ijass.2017.18.3.414.

10. Okuizumi, H., Sawada, H., Nagaike, H., Konishi, Y. \& Obayashi, S. Introduction of 1-m MSBS in Tohoku University, New Device for Aerodynamics Measurements of the Sports Equipment. Proceedings, 2, 273 (2018). https://doi.org/10.3390/proceedings2060273.

11. Linthorne, N. Design and materials in athletics. In Materials in Sports Equipment, 2nd ed.; Subic, A.; Woodhead Publishing: Cambridge, UK, 2, pp. 296-320 (2007). 
12. Kohama, Y. \& Kobayashi, R. Tohoku University Low-Turbulence Wind Tunnel, AIAA Paper, AIAA-19923913 (1992).

13. Hubbart, M. \& Bergman, C. Effect of Vibrations on Javelin Lift and Drag. J. appl. biomech., 5, 40-59 (1989). https://doi.org/10.1123/ijsb.5.1.40.

14. Maeda, M., Nomura, H., Shamoto, E., Moriwaki, T. \& Yanagida, Y. Dynamic Characteristics of Javelin and Athletic Performance. J. of Japan Soc. of Sports Industry, 3, 15-20 (1993). https://doi.org/10.5997/sposun.3.2_15

15. Seo, K., Shimoyama, K., Ota, K., Ohgi, Y. \& Kimura, Y. Optimization of flight distance and robustness in discus. Sports Eng., 18, 55-65 (2015). https://doi.org/10.1007/s12283-014-0166-y

16. Seo, K.; Watanabe, I.; Murakami, M. Optimal flight of a V-style ski jumping. Sports Eng., 7, 97-103 (2004).

\section{Figures}

\section{Figure 1}

A schematic of the magnetic suspension and balance system.

\section{Figure 2}

Commercially available women's javelin: (a) The javelin was spray painted white and a $15 \mathrm{~mm}$-long collar was attached to the javelin at the center of gravity; (b) Neodymium magnets were inserted along the longitudinal axis. (c) A 15mm-long-collar and grip string.

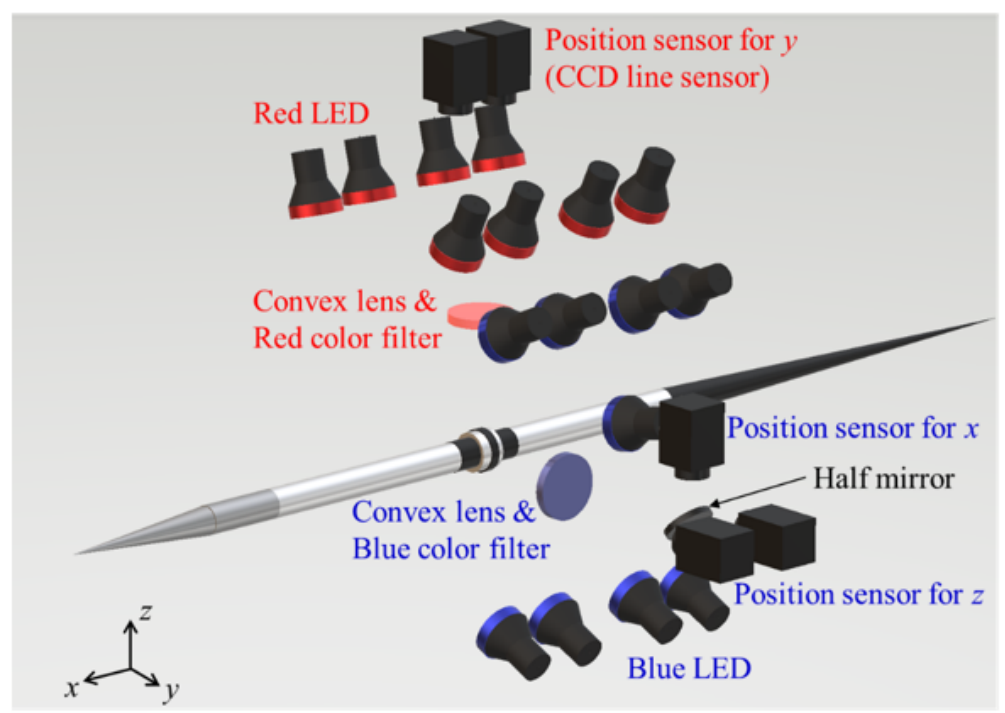

(a)

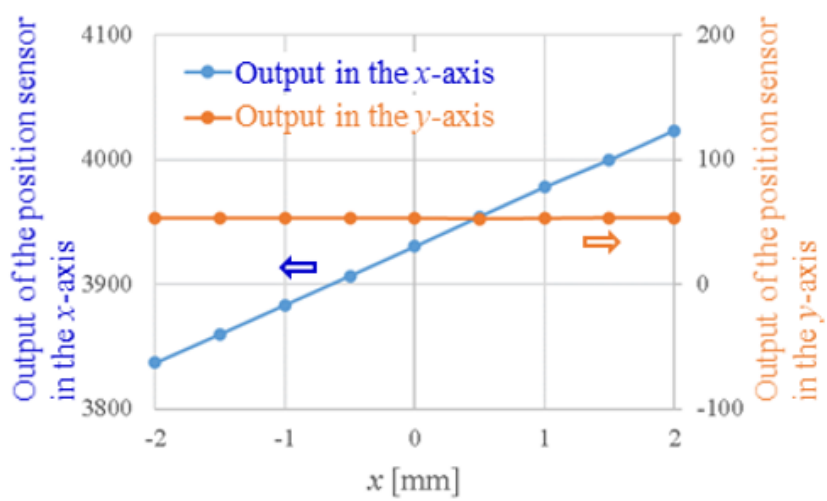

(b) 


\section{Figure 3}

The position-sensing system: (a) Schematic of the position-sensing system; (b) An example of the calibration results of position sensor in the $x$-axis.
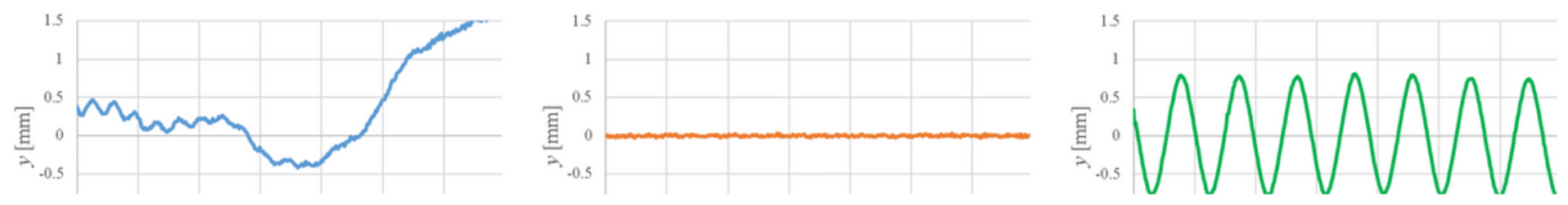

\section{Figure 4}

Time variations of the center of gravity of the javelin in the y-direction: (a) without the notch filter; (b) with the notch filter; (c) with a weak notch filter.

\section{Figure 5}

Javelin at $18^{\circ}$ in the magnetic suspension and balance system.

\section{Figure 6}

Force calibration in the x-axis: (a) Schematic; (b) Calibration result.

\section{Figure 7}

Meshes: (a) around the javelin; (b) around the javelin's top.

\section{Figure 8}


Aerodynamic coefficients as a function of the angle of attack, $A \circ A$. The static data are shown by open circles, while the dynamic (vibration) data are shown by open triangles. The $95 \%$ confidence intervals are also shown as error bars: (a) Drag coefficient, $C_{D}$ (b) Drag coefficient, $C_{L^{\prime}}$ (c) Pitching moment coefficient, $c_{m}$

\section{Figure 9}

EFD (Experimental Fluid dynamics) results by MSBS are shown by open circles, while the CFD results are shown by the solid line as a function of the angle of attack, AoA. The $95 \%$ confidence intervals are also shown as error bars: (a) Drag coefficient, $C_{D^{\prime}}$ (b) Drag coefficient, $C_{L^{;}}$(c) Pitching moment coefficient, $C_{m}$.

\section{Figure 10}

The pitching moment coefficient, $C_{m}$, obtained by CFD for the three cases. 